\title{
Vibro-tactile and visual asynchronies: Sensitivity and consistency
}

\author{
Jan B F van Erp, Peter J Werkhoven \\ TNO Human Factors, PO Box 23, NL 3769 ZG Soesterberg, The Netherlands; \\ e-mail:vanerp@tm.tno.nl \\ Received 12 November 2002, in revised form 14 August 2003
}

\begin{abstract}
We investigated the consistency between tactually and visually designated empty time intervals. In a forced-choice discrimination task, participants judged whether the second of two intervals was shorter or longer than the first interval. Two pulses defined the intervals. The pulse was either a vibro-tactile burst presented to the fingertip, or a foveally presented white square. The comparisons were made for uni-modal and cross-modal intervals. We used four levels of standard interval durations in the range of $100-800 \mathrm{~ms}$.

The results showed that tactile empty intervals must be $8.5 \%$ shorter to be perceived as long as visual intervals. This cross-modal bias is larger for small intervals and decreases with increasing standard intervals. The Weber fractions (the threshold divided by the standard interval) are $20 \%$ and are constant over the standard intervals. This indicates that the Weber law holds for the range of interval lengths tested. Furthermore, the Weber fractions are consistent over uni-modal and cross-modal comparisons, which indicates that there is no additional noise involved in the cross-modal comparisons.
\end{abstract}

\section{Introduction}

Because of growing complexity of visual interfaces, system designers are increasingly looking toward the auditory and/or tactile channels to provide an alternative or supplementary means of information transfer (van Erp 2001; Spence and Driver 1997). Effective multimodal interfaces require that stimulation from several sensory channels be coordinated and made congruent informationally as well as temporally (Kolers and Brewster 1985). In this experiment we investigated the uni-modal and cross-modal perception of empty visual and tactile intervals.

\subsection{Cross-modal bias}

An interesting question is how empty interval length is internally represented. For empty intervals it is tempting to assume that internal interval length is measured as the time between a particular feature of the response function (eg its onset, point of maximum curvature, or peak) to the first pulse and that same feature of the second pulse (eg the onsets of both pulses). In this case, the internal representation of interval length is independent of the shape of the response function or the particular feature used, and therefore independent of the modality, as long as the first marker does not affect the timing of the second. Such internal representation should not yield biases between modalities.

However, an alternative assumption (see also Grondin's internal marker hypothesisGrondin 1993) is that the internal representation is the time between one feature of the response function to the first pulse and another feature of the response function to the second pulse (eg the end of the first pulse and the onset of the second). In this case, internal interval length becomes dependent on the shape of the response functions and thus the modality of stimulation. First of all, response functions may be asymmetric in that the steepness of the response function at the onset of a pulse differs from its steepness at the offset. Second, the width of the response functions may differ for different modalities. This will result in a bias in cross-modal comparisons. 
The relationship between the auditory, visual, and tactile channels regarding temporal duration has not been studied extensively. Only the perceived durations of visual and auditory time intervals have been compared. For filled temporal intervals in the order of $1 \mathrm{~s}$, visual intervals had to be set longer than auditory intervals to be judged as equal in duration (Behar and Bevan 1961; Goldstone et al 1959; Goldstone and Goldfarb 1963; Goldstone and Lhamon 1972; Wearden et al 1998). The same crossmodal bias is present for empty intervals. The intervals bounded by light flashes, appear shorter than those bounded by brief auditory stimuli (Goldstone and Lhamon 1971; Sebel and Wilsoncroft 1983; Walker and Scott 1981).

Modality differences have also been reported for other time-related measures and tasks, for example in duration discrimination (Lhamon and Goldstone 1974), temporalorder judgment (Kanabus et al 2002), stimulus sequence identification (Garner and Gottwald 1968; Handel and Buffardi 1969), in the perception of temporal rhythms (Gault and Goodfellow 1938), and in a temporal-tracking and continuation-tapping task (Kolers and Brewster 1985).

We know of only two studies that have addressed auditory-tactile interval-duration comparisons. Both Ehrensing and Lhamon (1966), and Hawkes et al (1977) found perceived tactile durations to equal auditory ones. To our knowledge, no data are available for tactile - visual comparisons.

Based on the biased auditory-visual relation and the unbiased auditory-tactile relation, we expected a bias in tactile-visual comparisons as well: visual intervals will be set longer than tactile intervals to be judged as equal in duration. On the other hand, as manual interactions with the environment are often controlled through visual feedback, it may be expected that the perception of time intervals for the eye and for the fingertips has evolved to be consistent. Evidence for this comes from the development of visualhaptic interactions in children (Birch and Lefford 1963, 1967; see also Freides 1974).

\subsection{Cross-modal sensitivity}

Uni-modal threshold studies have shown that the temporal resolution of the skin lies between those of hearing and vision (Kirman 1973). This relation goes for numerous time-related measures and tasks, including discrimination of duration (Goodfellow 1934); synchronisation of fingertaps (Kolers and Brewster 1985) and adjusting empty intervals to equal pulse duration (Craig 1973). However, in their classic temporal-order study, Hirsh and Sherrick (1961) found that the thresholds (75\% correct) for the visual, auditive, and tactile modality were the same and in the order of $20 \mathrm{~ms}$. Although the temporal resolution of the skin has been investigated in several ways [eg with the fusion threshold (Geldard and Sherrick 1971; Gescheider 1966, 1967, 1974) and the gap detection performance (Van Doren et al 1990; Formby et al 1992)], tactile-interval discrimination has not yet been systematically studied as a function of interval length. Only Goodfellow (1934; see also Fraisse 1978, page 219) reported a difference threshold for interval duration of $9.5 \%$ (averaged across various methods used) for a standard interval of $1 \mathrm{~s}$.

To be able to compare visual information to tactile information in a cross-modal setting, there must be a common representation of the information from both senses. Several mechanisms for cross-modal visual-haptic comparisons have been suggested, based on two fundamentally different models (for an overview, see Summers and Lederman 1990). The first is based on modality-specific representations that are used for uni-modal comparisons (eg see Lederman et al 1990). These modality-specific representations must be translated into a common representation for cross-modal comparisons. This implies that cross-modal comparisons require an extra translation as compared to uni-modal comparisons. On the basis of the assumption that this extra translation increases the variability in the judgments, this model predicts a lower sensitivity for cross-modal comparisons than for uni-modal comparisons. 
The second model (Ernst 2001, page 88; see also Ernst and Banks 2002) states that information from the different modalities is directly processed and translated into a common representation. This representation is used for both uni-modal and cross-modal comparisons. In the latter model uni-modal and cross-modal comparisons are based on the same representation and are therefore hypothesised to have the same sensitivity.

\subsection{Objectives of our research}

A first objective was to determine the consistency (ie the cross-modal bias) of the perception of empty time intervals between the visual and tactile sensory modalities: are tactile intervals judged longer than visual, as is the case with auditory intervals? A second objective of this study was to determine the human sensitivity to discriminate empty intervals as a function of interval length. Finally, we wanted to compare crossmodal sensitivity to uni-modal sensitivity.

\section{Method}

\subsection{Participants}

Eight male, right-handed volunteers aged between 21 and 25 years, participated. They all reported normal vision and normal sense of touch. They were paid the equivalent of US \$40 for their participation.

\subsection{Stimulus and apparatus}

An empty interval was defined as the time between the onsets of two pulses of equal duration $(33.3 \mathrm{~ms}$ ). A visual pulse was a white square (subtending $5.1 \mathrm{deg} \times 5.1 \mathrm{deg}$ of visual angle) drawn on a monitor (Iiyama CRT with short persistence phosphor P22) against a dark background (for comparable stimuli, see Wearden et al 1998). The tactile pulse was presented by a Special Instruments Minivib 4 vibrator with a contact area of $22 \mathrm{~mm}$ by $16 \mathrm{~mm}$, which was attached to the skin with sticky tape and driven by a $250 \mathrm{~Hz}$ sine-wave signal (for comparable apparatus, see for example Mahar et al 1994). The amplitude of the vibration was about $30 \mathrm{~dB}$ above absolute threshold. The intensities of the tactile and visual stimulus were matched by a simple procedure (method of adjustment). Three observers adjusted the brightness of the visual stimulus ( 5 ascending and 5 descending trials per observer) so that the intensities were judged to be equal to the tactile stimulus. Since intensity of the markers does not affect cross-modal interval discrimination (Grondin et al 1996), the intensity of the visual stimulus was fixed at the mean value for these three observers.

A visual interval (V) was always presented foveally to both eyes. A tactile interval (T) was presented to the tip of the left index finger. The participants wore headsets with white noise that attenuated the sound of the vibrator to a subthreshold level during all conditions.

On each trial, two intervals were presented: a standard interval (S) and a comparison interval (C). Standard and comparison intervals were presented in random order. The pause between the presentation of the two intervals was $2 \mathrm{~s}$. Participants were asked to indicate whether the second interval was shorter or longer than the first interval by pushing the left or right mouse button. No feedback was given. The standard interval was $100,200,400$, or $800 \mathrm{~ms}$. The range of comparison intervals was chosen on the basis of pilot data, so that the percentage of trials to which the comparison interval was judged 'longer' than the standard ranged from $0 \%$ to $100 \%$. For each standard (except for the $100 \mathrm{~ms}$ standard), we chose to measure five longer comparison intervals and five shorter intervals. Equal step sizes were used between the comparisons and the $60 \mathrm{~Hz}$ refresh rate of the monitor was taken into account; this resulted in step sizes of $16.7,16.7,33.3$, and $66.7 \mathrm{~ms}$ for the 100, 200, 400, and $800 \mathrm{~ms}$ standard intervals, respectively. The values of standard and comparison intervals are given in table 1 . 
Table 1. Range and step size of the comparison intervals as function of the standard interval length.

Standard interval length/ms Range of the comparison intervals $/ \mathrm{ms} \quad$ Step size $/ \mathrm{ms}$

\begin{tabular}{lrl}
\hline 100.0 & $50.0-150.0$ & 16.7 \\
200.0 & $116.7-283.3$ & 16.7 \\
400.0 & $233.3-566.7$ & 33.3 \\
800.0 & $466.7-1133.3$ & 66.7 \\
\hline
\end{tabular}

\subsection{Marker-type conditions}

We tested temporal interval discrimination for four marker-type conditions: uni-modal discrimination ( $\mathrm{T}-\mathrm{T}$ and $\mathrm{V}-\mathrm{V}$ ) and cross-modal discrimination $[\mathrm{T}-\mathrm{V}$ (a standard tactile interval with a varying visual interval) and $\mathrm{V}-\mathrm{T}$ (a standard visual interval with a varying tactile interval)].

\subsection{Data fitting}

A method of constant stimuli was used testing 30 repetitions for each standard-comparison interval combination. We fitted a cumulative normal function to the fractions of longer responses for the range of comparison intervals, by MATHEMATICA ${ }^{\circledR}$ 3.0. The fit resulted in the point of subjective equality (PSE), and the threshold, defined as the increment of the comparison value relative to the PSE, for which the percentage of trials to which the comparison interval was judged longer than the standard was $75 \%$. The difference between the PSE and the standard interval was called bias.

\subsection{Design and statistical analyses}

The experiment consisted of four sessions. Each session tested a single standard interval. The four marker-type conditions were blocked within the session. The order of the sessions and the order of the marker types within a session were semi-balanced across participants. For each comparison interval, 30 repetitions were recorded.

Before analysis, the bias and threshold were standardised by dividing them by the standard interval. For the threshold, this results in the Weber fraction (Fechner 1860; Luce and Galanter 1963). The results were analysed with a repeated-measures analysis of variance (ANOVA): marker-type condition (4) $\times$ standard interval (4). Significant effects were further analysed by an a posteriori Tukey HSD test with $\alpha$ set at 0.05 .

\subsection{Procedure}

The participants were seated $90 \mathrm{~cm}$ in front of a monitor in a dimly lit room with their right hand on a computer mouse. They wore a sound-attenuating headset with white noise during all sessions to prevent hearing the faint humming sound of the vibrator.

At each trial, participants were asked to compare the length of two empty intervals and indicate whether the second interval was shorter or longer (forced choice) by pressing the left or right mouse button, respectively. The participant started the next trial by pressing the middle mouse button. There were four sessions, each one testing a specific standard interval. There were pauses in between sessions. Each participant completed the experiment on a single day.

\section{Results}

\subsection{Standardised bias}

The ANOVA on the standardised bias resulted in three significant effects: marker-type condition $\left(F_{3,21}=14.59, p<0.001\right)$, standard interval $\left(F_{3,21}=3.17, p<0.05\right)$, and the interaction between marker-type condition and standard interval $\left(F_{9,63}=4.82, p<0.001\right)$.

The interaction of marker-type condition and standard interval is depicted in figure 1 (aggregated over subjects). A positive bias is the result of observers judging the second 


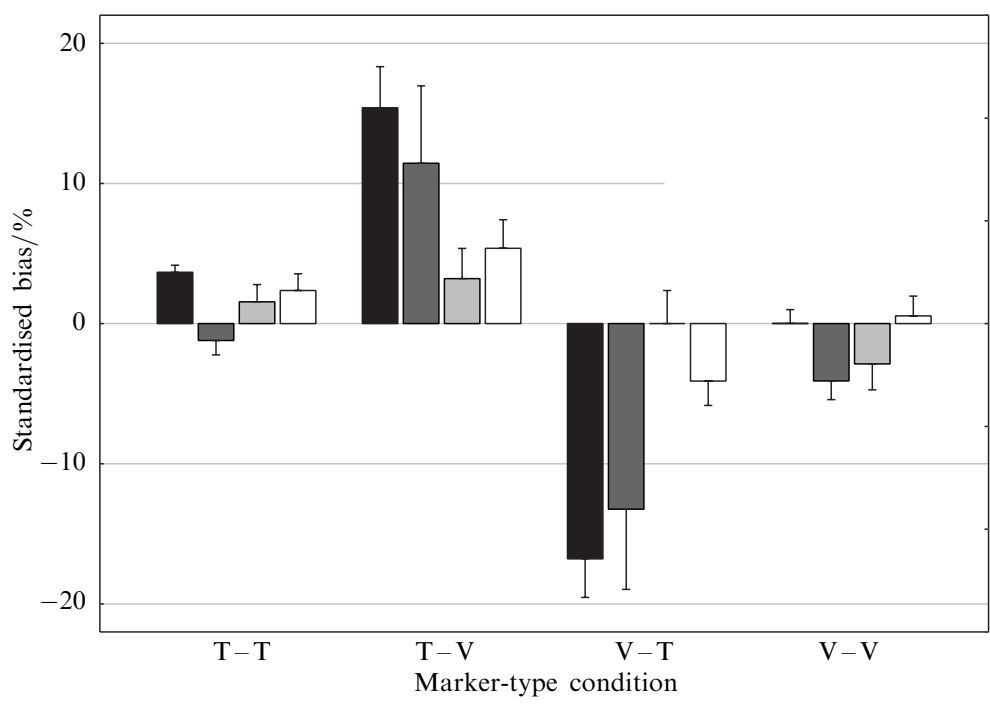

Standard interval:

$100 \mathrm{~ms}$ $200 \mathrm{~ms}$ $400 \mathrm{~ms}$ $800 \mathrm{~ms}$

Figure 1. The interaction between marker-type condition and standard interval length on the standardised bias. Marker-type conditions indicate the modalities (tactile, T; and visual, V) of the standard interval and the comparison interval, respectively. Positive values indicate that the comparison interval is longer than the standard interval. Bars indicate $1 \mathrm{SE}$.

marker-type condition as longer. The a posteriori test on the interaction can best be summarised as follows. The standardised bias in the cross-modal conditions with the smaller standard intervals (100 and $200 \mathrm{~ms}$, ie 4 means in total) is significantly higher than in the other conditions (12 means).

\subsection{Weber fractions}

The ANOVA on the Weber fraction resulted in one significant effect: the interaction between marker-type condition and standard interval $\left(F_{9,63}=4.61, p<0.001\right)$. This interaction is depicted in figure 2. The a posteriori analysis of this effect showed no specific pattern of significant differences.

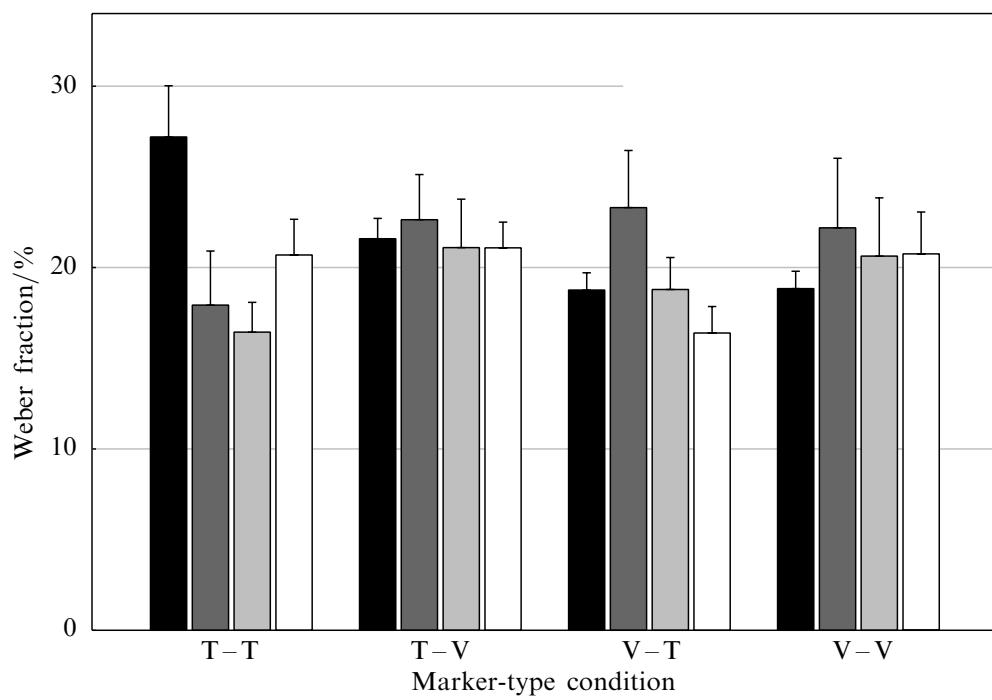

Standard interval:

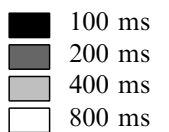

Figure 2. The interaction between marker-type condition and standard interval length on the Weber fraction. Marker-type conditions (tactile, T; and visual, V) indicate the modalities of the standard interval and the comparison interval, respectively. Bars indicate $1 \mathrm{SE}$. 


\section{Discussion}

\subsection{Bias}

In principle, no bias is expected when the two intervals to be compared are of the same type, that is, presented in the same modality $(\mathrm{T}-\mathrm{T}$ and $\mathrm{V}-\mathrm{V})$. The two intervals to be compared differ only in their interval length, not in any other stimulus dimension. Further, because the order of presentation was randomised, any preference for judging the second interval as shorter or longer will have an effect on the threshold, but not on the bias.

However, the data show a small bias for these same-type discriminations. In order to perceive the comparison interval as equally long as the standard interval, the comparison interval has to be $2.3 \%$ longer on average (independently of interval length). This is probably due to the fact that the stimulus dimension 'interval length' is not symmetric, but stretches from zero to infinity. In contrast, the cumulative normal distribution that is fitted along this stimulus dimension stretches from -infinity to +infinity. When a symmetric curve is fitted to such asymmetric data, the PSEs will be systematically raised relative to the standard value (a positive bias) as observed in this experiment. We also fitted the data after a log transform of the time parameter. Although this resulted in a smaller bias, the improvement was not statistically significant.

Of course, the same effect may bias the PSEs in a positive direction for marker-type conditions where intervals of different type (presented in different modalities) have to be discriminated. In fact, applying the $2.3 \%$ correction to the $\mathrm{T}-\mathrm{V}$ and $\mathrm{V}-\mathrm{T}$ data results in a bias of $8.8 \%$ and $8.5 \%$, respectively. This suggests that the length of a visual interval is systematically underestimated compared to a tactile interval, and/or that the length of a tactile interval is systematically overestimated compared to a visual interval, which confirms our hypothesis, based on the biased auditory-visual relation and the unbiased auditory - tactile relation. The interaction of marker-type condition and standard interval length shows that this bias is only present for short intervals. We will discuss two hypotheses for this bias.

\subsection{Internal-marker hypothesis}

Grondin's internal-marker hypothesis (Grondin 1993) is that the internal representation is the time between one feature of the response function to the first pulse and another feature of the response function to the second pulse. Consequently, a comparison between tactile and visual intervals may yield biases caused by the differences in response functions. For example, tactile response functions in the fingertip may ascend more steeply, and/or descend faster compared with visual-response functions. In that case, the time between the internal representations of the offset of the first pulse and the onset of the second is longer for a tactile interval than for a visual interval. Consequently, tactile intervals will be overestimated in length. This hypothesis predicts that a cross-modal bias is dependent on the response functions only and independent of the interval length. To test this, we performed an ANOVA on the raw (not standardised) bias. This analysis showed a highly significant effect of standard interval $\left(F_{3,21}=11686.94, p<0.001\right)$. The means were $6.2,12.1,24.7$, and $49.6 \mathrm{~ms}$ for a standard interval of 100, 200, 400, and $800 \mathrm{~ms}$, respectively. This result argues against the internalmarker hypothesis that predicts a constant bias that is independent of the length of the interval.

\subsection{Directed-attention hypothesis}

A second explanation for the cross-modal bias is based on the effect of directed attention on time-related tasks. For instance, Stelmach and Herdman (1991), Carver and Brown (1997), and Spence et al (2001) showed the effect of directed attention on the perception of temporal order and simultaneity. The attended stimulus appears to occur before the unattended stimulus when both are presented simultaneously. Recent data indicate 
that directed attention also has an effect on the perceived duration of a brief stimulus. Mattes and Ulrich (1998) found that stimuli (stimulus durations between 70 and $270 \mathrm{~ms}$ ) in the attended modality (either visual or auditory) were rated as longer than stimuli in the unattended modality. This effect of attention on brief stimuli was recently confirmed by Chen and O'Neill (2001). If, in a cross-modal condition, there is an asymmetry in the capture of attention or in the shifting of attention between the modalities, biases in perceived duration may occur. The interval presented in the modality that captures or holds attention will be judged as longer. Spence and McGlone (2001) recently showed that tactile spatial attention can be reflexively directed toward peripheral tactile stimuli. Comparisons between the costs involved (in terms of required time) when switching attention between modalities (auditory, visual, and tactile) were made by Spence and Driver (1997). They indeed found consistently higher costs of shifting from the tactile to the visual modality than vice versa. These asymmetrical effects between the visual and tactile channels combined with the effect of attention on duration perception (especially for brief stimuli) may explain the cross-modal bias and the effect of an increased bias for smaller interval lengths. However, both effects must be investigated further, for example by using a directed-attention paradigm.

\subsection{Sensitivities}

The sensitivities (ie the $75 \%$ correct threshold of interval discrimination) are most easily discussed in terms of Weber fractions since the results show that these are invariant with standard interval. A Weber law (the sensitivity is a constant fraction of the interval length) seems to hold for the range of standard intervals tested $(100-800 \mathrm{~ms})$. Our Weber fractions $(20 \%)$ seem to be in between the results reported by Grondin (1993, page 386 on $250 \mathrm{~ms}$ empty visual-interval discrimination) $\left(\Delta t_{75}=23 \%\right.$ ) and those of Grondin (1998), and McKee and Taylor (1984) who reported Weber fractions around $10 \%$ for the visual modality.

\subsection{Consistency of cross-modal sensitivities}

Participants estimate only two types of intervals: visual presentation in the fovea and tactile on the fingertip. The Weber fraction is $20.6 \%$ in the uni-modal marker-type conditions, and $20.5 \%$ in the cross-modal marker-type conditions. This confirms the predictions of a cross-modal comparison model in which the same (common) representation is used for both the uni-modal and cross-modal comparisons. This indicates that empty interval length is an amodal invariant (Lewkowicz 2002) for the visual and tactile sensory system.

\section{Conclusions}

The present experiment on tactile and visual discrimination of empty temporal intervals can be summarised as follows. The length of tactile intervals is systematically overestimated compared to visually presented intervals, and/or the length of visual intervals is underestimated compared to tactile intervals. This bias decreases for larger interval lengths. Within the range $100-800 \mathrm{~ms}$, Weber fractions for empty interval discrimination (tactile, visual, and cross-modal) are in the order of $20 \%$. The Weber fractions are constant over the interval range tested (ie the Weber law holds), and the Weber fractions are consistent over the uni-modal and cross-modal marker-type conditions.

\section{References}

Behar I, Bevan W, 1961 "The perceived duration of auditory and visual intervals: Cross-modal comparison and interaction" American Journal of Psychology 74 17-26

Birch H G, Lefford A, 1963 "Intersensory development in children" Monographs of the Society for Research in Child Development 28 (5, No. 89) pp 1-47

Birch H G, Lefford A, 1967 "Visual differentiation, intersensory integration and voluntary motor control" Monographs of the Society for Research in Child Development 32 (2, No. 110) pp 1-82 
Carver R A, Brown V, 1997 "Effects of amount of attention allocated to the location of visual stimulus pairs on perception of simultaneity" Perception \& Psychophysics 59 534-542

Chen Z, O'Neill P, 2001 "Processing demand modulates the effects of spatial attention on the judged duration of a brief stimulus" Perception \& Psychophysics 63 1229-1238

Craig J, 1973 "A constant error in the perception of brief temporal intervals" Perception \& Psychophysics 13 99-104

Craig J C, Baihua X, 1990 "Temporal order and tactile patterns" Perception \& Psychophysics 47 $22-34$

Ehrensing R H, Lhamon W T, 1966 "Comparison of tactile and auditory time judgments" Perceptual and Motor Skills 23 929-930

Ernst M O, 2001 Psychophysikalische Untersuchungen zur visuomotorischen Integration beim Menschen: Visuelle und haptische Wahrnehmung virtueller und realer Objekte (Tübingen, Germany: Medien Verlag Köhler)

Ernst M O, Banks M S, 2002 "Humans integrate visual and haptic information in a statistically optimal fashion" Nature 415429 - 433

Erp J B F van, 2001 "Tactile navigation display", in Haptic Human-Computer Interaction Eds S Brewster, R Murray-Smith, Lecture Notes in Computer Science (Berlin: Springer) pp $165-173$

Fechner G T, 1860 Elemente der Psychophysik (Leipzig: Breitkopf \& Härtel)

Formby C, Morgan L N, Forrest T G, Raney J J, 1992 "The role of frequency selectivity in measures of auditory and vibrotactile temporal resolution" Journal of the Acoustical Society of America 91293 - 305

Fraisse P, 1978 "Time and rhythm perception", in Handbook of Perception VIII Eds E Carterette, M Friedman (New York: Academic Press) pp 203-254

Freides D, 1974 "Human information processing and sensory modality: cross-modal functions, information complexity, memory, and deficit" Psychological Bulletin 81284 -310

Garner W R, Gottwald R L, 1968 “The perception and learning of temporal patterns" Quarterly Journal of Experimental Psychology $2097-107$

Gault R H, Goodfellow L D, 1938 "An empirical comparison of audition, vision, and touch in the discrimination of temporal patterns and ability to reproduce them" Journal of General Psychology $1841-47$

Geldard F A, Sherrick C E, 1971 "Cutaneous temporal acuity" Princeton Cutaneous Research Project (PCRP) Report 18 Princeton University, Princeton, NJ

Gescheider G A, 1966 "The resolving of successive clicks by the ears and skin" Journal of Experimental Psychology $71378-381$

Gescheider G A, 1967 "Auditory and cutaneous temporal resolution of successive brief stimuli" Journal of Experimental Psychology 75 570-572

Gescheider G A, 1974 "Temporal relations in cutaneous stimulation", in Cutaneous Communication Systems and Devices Ed. F A Geldard (Austin, TX: The Psychonomic Society) pp 33-37

Goldstone S, Boardman W K, Lhamon W T, 1959 "Intersensory comparisons of temporal judgments" Journal of Experimental Psychology $57243-248$

Goldstone S, Goldfarb J, 1963 "Judgment of filled and unfilled durations: Intersensory effects" Perceptual and Motor Skills $17763-774$

Goldstone S, Lhamon W T, 1971 "Levels of cognitive functioning and the auditory - visual difference in human timing behaviour", in Adaptation-level Theory: A Symposium Ed. M H Appley (New York: Academic Press) pp 263-280

Goldstone S, Lhamon W T, 1972 "Auditory-visual differences in human judgment" Perceptual and Motor Skills $34623-633$

Goodfellow L D, 1934 "An empirical comparison of audition, vision, and touch in the discrimination of short intervals of time" American Journal of Psychology 46 243-258

Grondin S, 1993 "Duration discrimination of empty and filled intervals marked by auditory and visual signals" Perception \& Psychophysics 54383 - 394

Grondin S, 1998 "Judgements of the duration of visually marked empty time intervals: Linking perceived duration and sensitivity" Perception \& Psychophysics $60319-330$

Grondin S, Irvy R B, Franz E, Perrault L, Metthe L, 1996 "Markers' influence on the duration discrimination of intermodal intervals" Perception \& Psychophysics 58 424-433

Handel S, Buffardi L, 1969 "Using several modalities to perceive one temporal pattern" Quarterly Journal of Experimental Psychology $21256-266$

Hawkes G R, Deardorff P A, Ray W S, 1977 "Response delay effects with cross-modality duration judgments" Journal of Auditory Research 17 55-57 
Hirsh I J, Sherrick C E, 1961 "Perceived order in different sense modalities" Journal of Experimental Psychology $62423-432$

Kanabus M, Szelag E, Rojek E, Poppel E, 2002 "Temporal order judgement for auditory and visual stimuli" Acta Neurobiologiae Experimentalis 62 263-270

Kirman J H, 1973 "Tactile communication of speech: A review and analysis" Psychological Bulletin $8054-74$

Kolers P A, Brewster J M, 1985 "Rhythms and responses" Journal of Experimental Psychology: Human Perception and Performance 11 150-167

Lederman S J, Klatzky R L, Chataway C, Summers C G, 1990 "Visual mediation and the haptic recognition of two-dimensional pictures of common objects" Perception \& Psychophysics 47 $54-64$

Lewkowicz D J, 2002 "Heterogeneity and heterochrony in the development of intersensory perception" Cognitive Brain Research 14 41-63

Lhamon W T, Goldstone S, 1974 "Studies of auditory - visual differences in human time judgment: 2. More transmitted information with sounds than lights" Perceptual and Motor Skills 39 $295-307$

Luce R D, Galanter E, 1963 "Discrimination", in Handbook of Mathematical Psychology Volume 1, Eds R D Luce, R R Bush, E Galanter (Chichester: John Wiley \& Sons) pp 191 - 243

McKee S P, Taylor D G, 1984 "Discrimination of time: comparison of foveal and peripheral sensitivity" Journal of the Optical Society of America A 1 620-627

Mahar D, MacKenzie B, McNicol D, 1994 "Modality-specific differences in the processing of spatially, temporally, and spatiotemporally distributed information" Perception 23 1369-1386

Mattes S, Ulrich R, 1998 "Directed attention prolongs the perceived duration of a brief stimulus" Perception \& Psychophysics $601305-1317$

Sebel A J, Wilsoncroft W E, 1983 "Auditory and visual differences in time perception" Perceptual and Motor Skills $57295-300$

Spence C, Driver J, 1997 "Cross-modal links in attention between audition, vision, and touch: Implications for interface design" International Journal of Cognitive Ergonomics 1351 -373

Spence C, McGlone F P, 2001 "Reflexive spatial orienting of tactile attention" Experimental Brain Research $141324-330$

Spence C, Shore D I, Klein R M, 2001 "Multisensory prior entry" Journal of Experimental Psychology: General $130799-832$

Stelmach L B, Herdman C M, 1991 "Directed attention and perception of temporal order" Journal of Experimental Psychology: Human Perception and Performance $17539-550$

Summers D C, Lederman S J, 1990 "Perceptual asymmetries in the somatosensory system: a dichhaptic experiment and critical review of the literature from 1929 to 1986" Cortex 26 $201-226$

Van Doren C L, Gescheider G A, Verillo R T, 1990 "Vibro-tactile temporal gap detection as a function of age" Journal of the Acoustical Society of America $872201-2206$

Walker J T, Scott K J, 1981 "Auditory-visual conflicts in the perceived duration of lights, tones, and gaps" Journal of Experimental Psychology: Human Perception and Performance 7 1327-1339

Wearden J H, Edwards H, Fakhri M, Percival A, 1998 "Why sounds are judged longer than lights: application of a model of the internal clock in humans" Quarterly Journal of Experimental Psychology $5197-120$ 


\section{PERCEPTION}

VOLUME 332004

www.perceptionweb.com

Conditions of use. This article may be downloaded from the Perception website for personal research by members of subscribing organisations. Authors are entitled to distribute their own article (in printed form or by e-mail) to up to 50 people. This PDF may not be placed on any website (or other online distribution system) without permission of the publisher. 